
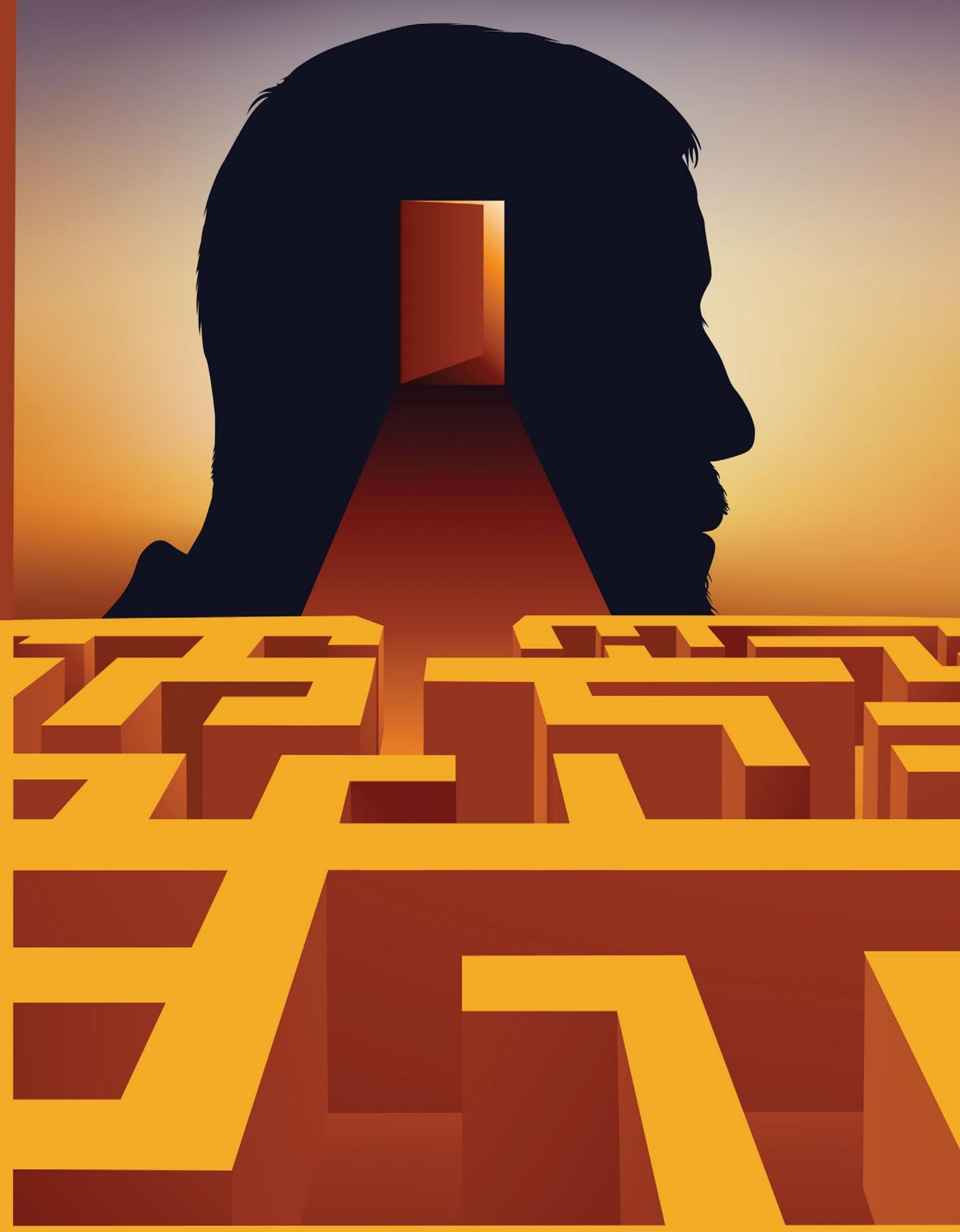


\section{Early interventions for psychosis}

\section{Despite considerable challenges, prevention and first-episode recovery programs may improve outcomes}

\author{
Morris B. Goldman, MD \\ Associate Professor of Psychiatry and \\ Behavioral Sciences
}

\section{Marko Mihailovic, MA, LCPC \\ Clinical Research Manager}

\section{Philip G. Janicak, MD}

Adjunct Professor of Psychiatry and Behavioral Sciences

Northwestern University

Chicago, Illinois

\section{Disclosures}

Dr. Goldman has served as a speaker for PsychU/Otsuka, received research grants from the National Institute of Mental Health, and received income from Sunovion. Mr. Mihailovic reports no financial relationships with any companies whose products are mentioned in this article, or with manufacturers of competing products. Dr. Janicak has served as a consultant to TMS Solutions and Neuronetics and as a speaker for PsychU/Otsuka.

doi: $10.12788 /$ cp.0176

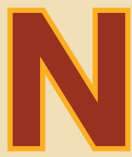

euroscience research over the past half century has failed to significantly advance the treatment of severe mental illness. ${ }^{1,2}$ Hence, evidence that a longer duration of untreated psychosis (DUP) aggravates-and early intervention with medication and social supports ameliorates - the long-term adverse consequences of psychotic disorders generated a great deal of interest. ${ }^{3,4}$ This knowledge led to the development of diverse early intervention services worldwide aimed at this putative "critical window." It raised the possibility that appropriate interventions could prevent the long-term disability that makes chronic psychosis one of the most debilitating disorders. ${ }^{5,6}$ However, even beyond the varied cultural and economic confounds, it is difficult to assess, compare, and optimize program effectiveness. ${ }^{7}$ Obstacles include paucity of sufficiently powered, well-designed randomized controlled trials (RCTs), the absence of diagnostic biomarkers or other prognostic indicators to better account for the inherent heterogeneity in the population and associated outcomes, and the absence of modifiable risk factors that can guide interventions and provide intermediate outcomes. ${ }^{4,-10}$

To better appreciate these issues, it is important to distinguish whether a program is designed to prevent psychosis, or to mitigate the effects of psychosis. Two models include the:

- Prevention model, which focuses on young individuals who are not yet overtly psychotic but at high risk

- First-episode recovery model, which focuses on those who have experienced a first episode of psychosis (FEP) but have not yet developed a chronic disorder. 


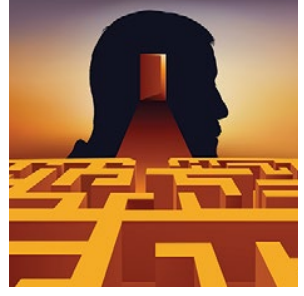

\section{Preventing psychosis}

\section{Clinical Point}

Initial meta-analyses reported that CBT and antipsychotics prevented one-half of individuals from becoming psychotic at 12 months

Discuss this article at www.facebook.com/ MDedgePsychiatry

continued from page 13

Both models share long-term goals and are hampered by many of the same issues summarized above. They both deviate markedly from the standard medical model by including psychosocial services designed to promote restoration of a self-defined trajectory to greater independence. ${ }^{11-14}$ The 2 differ, however, in the challenges they must overcome to produce their sample populations and establish effective interventions. ${ }^{10,15,16}$

In this article, we provide a succinct overview of these issues and a set of recommendations based on a "strength-based" approach. This approach focuses on finding common ground between patients, their support system, and the treatment team in the service of empowering patients to resume responsibility for transition to adulthood.

\section{The prevention model}

While most prevention initiatives in medicine rely on the growing ability to target specific pathophysiologic pathways, ${ }^{3}$ preventing psychosis relies on clinical evidence showing that DUP and early interventions predict a better course of severe mental illness. ${ }^{17}$ In contrast, initiatives such as normalizing neonatal neuronal pathways are more consistent with the strategy utilized in other fields but have yet to yield a pathophysiologic target for psychosis. ${ }^{3,18}$

\section{Initial efforts to identify 'at-risk' individuals}

The prevention model of psychosis is based on the ability to identify young individuals at high risk for developing a psychotic disorder (Figure, page 25). The first screening measures were focused on prodromal psychosis (eg, significant loss of function, family history, and "intermittent" and "attenuated" psychotic symptoms). When applied to referred (ie, pre-screened) samples, 30\% to $40 \%$ of this group who met criteria transitioned to psychosis over the next 1 to 3 years despite antidepressant and psychosocial interventions. ${ }^{19}$ Comprising 8 academic medical centers, the North American Prodrome Longitudinal Study (NAPLS) produced similar results using the Structured Interview for Prodromal Syndromes (SIPS). ${ }^{17}$ Thus, $30 \%$ to $50 \%$ of pre-screened individuals referred by school counselors and mental health professionals met SIPS criteria, and 35\% of these individuals transitioned to psychosis over 30 months. The validity of this measure was further supported by the fact that higher baseline levels of unusual thought content, suspicion/paranoia, social impairment, and substance abuse successfully distinguished approximately $80 \%$ of those who transitioned to psychosis. The results of this first generation of screening studies were exciting because they seemed to demonstrate that highly concentrated samples of young persons at high risk of developing psychosis could be identified, and that fine-tuning the screening criteria could produce even more enriched samples (ie, positive predictive power).

\section{Initial interventions produced promising results}

The development of effective screening measures led to reports of effective treatment interventions. These were largely applied in a clinical staging model that restricted antipsychotic medications to those who failed to improve after receiving potentially "less toxic" interventions (eg, omega-3 polyunsaturated fatty acids and other antioxidants; psychotherapy; cognitive-behavioral therapy [CBT]; family therapy). ${ }^{5}$ While study designs were typically quasi-experimental, the interventions appeared to dramatically diminish the transition to psychosis (ie, approximately $50 \%$ ).

The first generation of RCTs appeared to confirm these results, although sample sizes were small, and most study designs assessed only a single intervention. Initial meta-analyses of these data reported that both CBT and antipsychotics appeared to prevent approximately one-half of individuals from becoming psychotic at 12 months, and more than one-third at 2 to 4 years, compared with treatment as usual. ${ }^{20}$

While some researchers challenged the validity of these findings, ${ }^{21-23}$ the results generated tremendous international enthusiasm and calls for widespread implementation. ${ }^{6}$ The number of early intervention services (EIS) centers increased dramatically worldwide, and in 2014 the National Institute for Health and Care Excellence released 


\section{Steps in identifying the population for prevention RCTs}

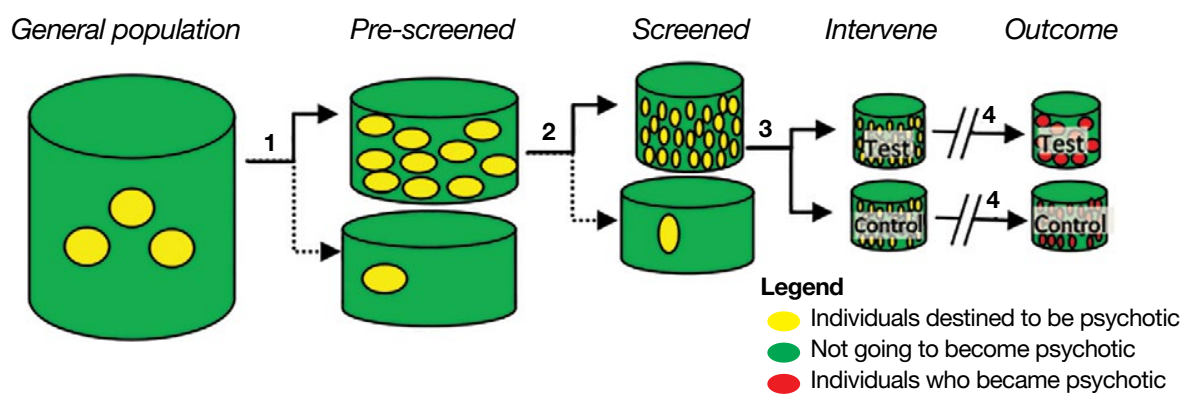

STEP 1 needs to produce an enriched Pre-screened sample (maximize yellow circles) (ideally containing $100 \%$ of those destined to be psychotic in nationwide programs) while eliminating as many of those not destined to become psychotic (minimize green volume).

STEP 2 produces Screened individuals at high risk of becoming psychotic by retaining as many as possible of the Pre-screened individuals destined to become psychotic (true positives) and eliminating as many as possible of those who will not (true negatives). Note the success of Step 2 is highly dependent on the success of Step 1 because the Pre-screened sample must not eliminate persons who will transition to psychosis while at the same time eliminating as many as possible of those who will not. If instead the false positives drown out the number of true positives in the Pre-screened sample, it will be very difficult to have a sufficiently enriched Screened high-risk sample to justify program expense and demonstrate program efficacy.

STEP 3: After randomizing participants to 2 groups, Control (treatment as usual) and Test, treatments are initiated (Intervene).

STEP 4: Program efficacy is determined by comparing the transition to psychosis in the 2 groups over the next several years (Outcome). Note how the presence of persons not destined to become psychotic in both groups limits the ability to detect true effects.

Within the relevant population of young adults (green volume), there are a small number of individuals destined to become psychotic over the next 2 to 3 years (yellow circles). For prevention programs, the goal is to identify this group and intervene to prevent their transition to psychosis (Outcome: Test vs Contro/). To determine whether these efforts are successful, a clinician must be able to identify a group containing a high percentage of individuals at very high risk of becoming psychotic (sensitivity) and a low percentage of individuals at very low risk of becoming psychotic (Screened).

For FEP programs, the sequence is similar, but Step 1 and Step 2 are much more tractable, while Step 4 presents greater challenges for FEP than prevention programs, intermediate outcomes have been better utilized. See the main article for further explanation.

FEP: first episode of psychosis; RCTs: randomized controlled trials

standards for interventions to prevent transition to psychosis. ${ }^{24}$ These included close monitoring, CBT and family interventions, and avoiding antipsychotics when possible. ${ }^{24}$

\section{Focusing on sensitivity over specificity}

The first generation of studies generated by the prevention model relied on outreach programs or referrals, which produced small samples of carefully selected, pre-screened individuals (Figure, Pre-screened) who were then screened again to establish the high-risk sample. ${ }^{25}$ While approximately $33 \%$ of these individuals became psychotic, the screening process required a very efficient means of eliminating those not at high-risk (given the ultimate target population represented only approximately $.5 \%$ of young people) (Figure). The pre-screening and screening processes in these first-generation studies were labor-intensive but could only identify approximately $5 \%$ of those individuals destined to become psychotic over the next 2 or 3 years. Thus, alternative methods to enhance sensitivity were needed to extend programming to the general population.

\section{Clinical Point}

The first generation
of studies of the
prevention model
produced small
samples of carefully
selected, pre-
screened individuals




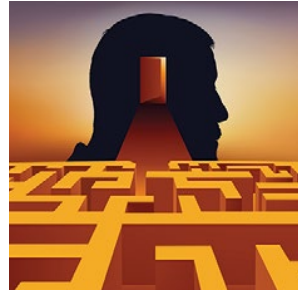

\section{Preventing psychosis}

\section{Clinical Point}

Diluting the pool of study participants with those not at risk makes it nearly impossible to identify effective interventions
Second-generation pre-screening (Figure; Step 1, page 25). New pre-screening methods were identified that captured more individuals destined to become psychotic. For example, approximately $90 \%$ of this population were registered in health care organizations (eg, health maintenance organizations) and received a psychiatric diagnosis in the year prior to the onset of psychosis (true positives). ${ }^{8}$ These samples, however, contained a much higher percentage of persons not destined to become psychotic, and somehow the issue of specificity (decreasing false positives) was minimized. ${ }^{8,9}$ For example, pre-screened samples contained 20 to 50 individuals not destined to become psychotic for each one who did. ${ }^{26}$ Since screening measures could only eliminate approximately $20 \%$ of this group (Figure, Step 2, page 25), second-generation transition rates fell from $30 \%$ to $40 \%$ to $2 \%$ to $10 \% .27,28$

Other pre-screening approaches were introduced, but they also focused on capturing more of those destined to become psychotic (sensitivity) than eliminating those who would not (specificity). For instance, Australia opened more than 100 "Headspace" community centers nationwide designed to promote engagement and self-esteem in youth experiencing anxiety; depression; stress; relationship, work, or school problems; or bullying. ${ }^{13}$ Most services were free and included mental health staff who screened for psychosis and provided a wide range of services in a destigmatized setting. These methods identified at least an additional $5 \%$ to $7 \%$ of individuals destined to become psychotic, but to our knowledge, no data have been published on whether they helped eliminate those who did not.

Second-generation screening (Figure, Step 2, page 25). A second screening aims to retain those pre-screened individuals who will become psychotic (ie, minimizing false negatives) while further minimizing those who do not (ie, minimizing false positives). The addition of cognitive, neural (eg, structural MRI; neurophysiologic), and biochemical (eg, inflammatory immune and stress) markers to the risk calculators have produced a sensitivity close to $100 \% \%^{8,9}$ Unfortunately, these studies downplayed specificity, which remained approximately
$20 \%{ }^{8,9}$ Specificity is critical not just because of concerns about stigma (ie, labeling people as pre-psychotic when they are not) but also because of the adverse effects of antipsychotic medications and the effects on future program development (interventions are costly and labor-intensive). Also, diluting the pool with individuals not at risk makes it nearly impossible to identify effective interventions (ie, power). ${ }^{27,28}$

While some studies focused on increasing specificity (to approximately $75 \%$ ), this leads to an unacceptable loss of sensitivity (from $90 \%$ to $60 \%){ }^{29}$ with $40 \%$ of pre-screened individuals who would become psychotic being eliminated from the study population. The addition of other biological markers (eg, salivary cortisol) $)^{30}$ and use of learning health systems may be able to enhance these numbers (initial reports of specificity $=87 \%$ and sensitivity $=85 \%)^{8,9}$ This is accomplished by integrating artificial and human intelligence measures of clinical (symptom and neurocognitive measures) and biological (eg, polygenetic risk scores; gray matter volume) variables. ${ }^{31}$ However, even if these results are replicated, more effective pre-screening measures will be required.

Identifying a suitable sample population for prevention program studies is clearly more complicated than for FEP studies, where one can usually identify many of those in the at-risk population by their first hospitalization for psychotic symptoms. The issues of false positives (eg, substanceinduced psychosis) and negatives (eg, slow deterioration, prominent negative symptoms) are important concerns, but proportionately far less significant.

\section{Prevention and FEP interventions}

Once a study sample is constituted, 1 to 3 years of treatment interventions are initiated. Interventions for prevention programs typically include CBT directed at attenuated psychosis (eg, reframing or de-catastrophizing unusual thoughts and minimizing distress associated with unusual perceptions); case management to facilitate personal, educational, and vocational goals; and family therapy in single or multi-group formats to educate one's support system about the 


\section{Identifying biomarkers of severe mental illness}

B

iomarkers and modifiable risk factors $^{4,9,10,41,43}$ are at the core of personalized medicine and its ultimate objective (ie, theragnostics). This is the ability to identify the correct intervention for a disorder based on a biomarker of the illness..$^{10,36}$ The inability to identify biomarkers of severe mental illness is multifactorial but in part may be attributable to "looking in all the wrong places." ${ }^{41} \mathrm{By}$ focusing on neural processes that generate psychiatric symptomatology, investigators are assuming they can bridge the "mind gap"1 and specifically distinguish between pathological, compensatory, or collateral measures of poorly characterized limbic neural functions. ${ }^{41}$

It may be more productive to identify a pathological process within the limbic system that produces a medical condition as well as the mental disorder. If one can isolate the pathologic limbic circuit activity responsible for a medical condition, one may be able to reproduce this in animal models and determine whether analogous processes contribute to the core features of the mental illness. Characterization of the aberrant neural circuit in animal models also could yield targets for future therapies. For example, episodic water intoxication in a discrete subset of patients with schizophrenia ${ }^{44}$ appears to arise from a stress diathesis produced by anterior hippocampal pathology that disrupts regulation of antidiuretic hormone, oxytocin, and hypothalamic-pituitary-adrenal axis secretion. These patients also exhibit psychogenic polydipsia that may be a consequence of the same hippocampal pathology that disrupts ventral striatal and lateral hypothalamic circuits. These circuits, in turn, also modulate motivated behaviors and cognitive processes likely relevant to psychosis. ${ }^{45}$ risk state and to minimize adverse familial responses. ${ }^{14}$ Many programs also include supported education or employment services to promote reintegration in age-appropriate activities; group therapy focused on substance abuse and social skills training; cognitive remediation to ameliorate the cognitive dysfunction; and an array of pharmacologic interventions designed to delay or prevent transition to psychosis or to alleviate symptoms. While most interventions are similar, FEP programs have recently included peer support staff. This appears to instill hope in newly diagnosed patients, provide role models, and provide peer supporters an opportunity to use their experiences to help others and earn income. ${ }^{32}$

The breadth and depth of these services are critical because retention in the program is highly dependent on participant engagement, which in turn is highly dependent on whether the program can help individuals get what they want (eg, friends, employment, education, more autonomy, physical health). The setting and atmosphere of the treatment program and the willingness/ ability of staff to meet participants in the community are also important elements. ${ }^{11,12}$ In this context, the Headspace community centers are having an impact far beyond Australia and may prove to be a particularly good model. ${ }^{13}$

\section{Assessing prevention and FEP interventions}

The second generation of studies of prevention programs has not confirmed, let alone extended, the earlier findings and meta-analyses. A 2020 report concluded CBT was still the most promising intervention; it was more effective than control treatments at 12 and 18 months, although not at 6,24 , or 48 months..$^{33}$ This review included controlled, open-label, and naturalistic studies that assessed family therapy; omega-3 polyunsaturated fatty acids; integrated psychological therapy (a package of interventions that included family education, CBT, social skills training, and cognitive remediation); $N$-methyl-Daspartate receptor modulators; mood stabilizers; and antipsychotics. In addition to the evidence supporting CBT, the results also indicated nonsignificant trends favoring family and integrated psychological therapy. Neither a 2019 Cochrane review ${ }^{34}$ nor a 2020 "umbrella" assessment of 42 meta-analyses ${ }^{9}$ found convincing evidence for the efficacy of any program components.

While these disappointing findings are at least partly attributable to the methodological challenges described above and in the Figure (page 25), other factors may hinder establishing effective interventions. In contrast to FEP studies, those focused on prevention had a very ambitious agenda

\section{Clinical Point}

The second generation of studies of prevention programs has not confirmed or extended the earlier findings 


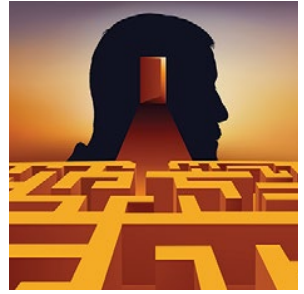

\section{Preventing psychosis}

\section{Clinical Point}

In contrast to FEP studies, those focused on prevention tended to downplay more modest intermediate outcomes (eliminating psychosis) and tended to downplay more modest intermediate outcomes. These studies also tended to assess new ideas with small samples rather than pursue promising findings with larger multi-site studies focused on a group of interventions. The authors of a Cochrane review observed "There is the impression that in this whole area there is a triumph of hope over adversity. There is the repeated hope invested in another-often uniquestudy question and then a study of fewer than 100 participants are completed. This results in the set of comparisons reported here, all 9 of which are too underpowered to really highlight clear differences." ${ }^{\prime 34}$ To use a baseball analogy, it seems that investigators are "swinging for the fence" when a few singles are what's really needed.

From the outset, the goals of FEP studies were more modest, largely ignoring the task of developing consensus definitions of recovery that require following patients for up to 5 to 10 years. Instead, they use intermediate endpoints based on adapting treatments that already appeared effective in patients with chronic mental disorders. ${ }^{35}$ As a consequence, researchers examining FEP demonstrated clear, albeit limited, salutary effects using large multi-site trials and previously established outcome measures. ${ }^{3,10,36}$ For instance, the Recovery After an Initial Schizophrenia Episode-Early Treatment Program (RAISEETP) study was a 2-year, multi-site RCT ( $\mathrm{N}=404)$ funded by the National Institute of Mental Health (NIMH). The investigators reported improved indices of social function (eg, quality of life; education and work participation) and total ratings of psychopathology and depression compared with treatment as usual. Furthermore, they established that DUP predicted treatment response. ${ }^{35}$ The latter finding was underscored by improvement being limited to the $50 \%$ with $<74$ weeks DUP. Annual costs of the program per 1 standard deviation improvement in quality of life were approximately $\$ 1,000$ for patients with $<74$ weeks DUP and $\$ 40,000$ for those with $>74$ weeks DUP. Concurrent meta-analyses confirmed and extended these findings, ${ }^{16}$ showing higher remission rates; diminished relapses and hospital admissions; greater engagement in programming; greater involvement in work and school; improved quality of life; and other steps toward recovery. These studies were also able to establish a clear benefit of antipsychotic medications, particularly a high acceptance of long-acting injectable antipsychotic formulations, which promoted adherence and decreased some adverse events $\mathrm{s}^{37}$; and early use of clozapine therapy, which improved remission rates and longer-term outcomes. ${ }^{38}$ Other findings underscored the need to anticipate and address new problems associated with effective antipsychotic therapy (eg, antipsychotic response correlates with weight gain, a particularly intolerable adverse event for this age group). ${ }^{39}$ Providing pre-emptive strategies such as exercise groups and nutritional education may be necessary to maintain adherence.

\section{Limitations of FEP studies}

The effect sizes in these FEP studies were small to medium on outcome measures tracking recovery and associated indicators (eg, global functioning, school/work participation, treatment engagement); the number needed to treat for each of these was $>10$. There is no clear evidence that recovery programs such as RAISE-ETP actually reduce longer-term disability. Most studies showed disability payments increased while clinical benefits tended to fade over time. In addition, by grouping interventions together, the studies made it difficult to identify effective vs ineffective treatments, let alone determine how best to personalize therapy for participants in future studies.

\section{The next generation of FEP studies} While limited in scope, the results of the recent FEP studies justify a next generation of recovery interventions designed to address these shortcomings and optimize program outcomes..$^{39}$ Most previous FEP studies were conducted in community mental health center settings, thus eliminating the need to transition services developed in academia into the "real world." The next generation of NIMH studies will be primarily conducted in analogous settings under the Early Psychosis Intervention Network (EPINET).$^{40}$ EPINET's 


\section{A strength-based approach to early interventions for psychosis}

\begin{tabular}{|c|c|c|}
\hline Recommendation & Example & Source \\
\hline $\begin{array}{l}\text { Avoid divisive aspects of the } \\
\text { medical model }\end{array}$ & "You must take medication" & Dixon et al ${ }^{35}(2018)$ \\
\hline $\begin{array}{l}\text { Seek common ground with } \\
\text { the patient }\end{array}$ & "What are you interested in?" & Dixon et al ${ }^{12}(2016)$ \\
\hline $\begin{array}{l}\text { Commit to helping the patient } \\
\text { reach their goals }\end{array}$ & "Let's think about next steps" & Tindall et a ${ }^{11}(2020)$ \\
\hline $\begin{array}{l}\text { Identify needed skills and } \\
\text { barriers to achievement }\end{array}$ & "Can you read a book?" & Dixon et al ${ }^{12}(2016)$ \\
\hline $\begin{array}{l}\text { Consider long-acting } \\
\text { injectable antipsychotics }\end{array}$ & $\begin{array}{l}\text { "I believe the shot may reduce tension by } \\
\text { getting your parents off your back" }\end{array}$ & Kane et al ${ }^{37}(2020)$ \\
\hline $\begin{array}{l}\text { Look to clozapine after first } \\
\text { failed antipsychotic }\end{array}$ & $\begin{array}{l}\text { "It's hard to pay attention in class when } \\
\text { you can't ignore the voices" }\end{array}$ & Morrison et al ${ }^{38}(2020)$ \\
\hline $\begin{array}{l}\text { Anticipate problems and } \\
\text { solutions }\end{array}$ & $\begin{array}{l}\text { "Would you be willing to meet with a } \\
\text { nutritionist and join an exercise group?" }\end{array}$ & Chen et al ${ }^{39}(2021)$ \\
\hline $\begin{array}{l}\text { Maintain a noncritical attitude } \\
\text { toward failure }\end{array}$ & "What have we learned?" & Tindall et a $\left.\right|^{11}(2020)$ \\
\hline $\begin{array}{l}\text { Consider EIS (if available) or } \\
\text { other resources }\end{array}$ & $\begin{array}{l}\text { "There is a local program you and your } \\
\text { family may want to check out" }\end{array}$ & $\begin{array}{l}\text { Early Assessment and } \\
\text { Support Alliance }{ }^{46}(2021)\end{array}$ \\
\hline $\begin{array}{l}\text { Consider attempting to } \\
\text { re-create key EIS elements }\end{array}$ & $\begin{array}{l}\text { "I know a therapist and counselor who } \\
\text { may help you find a job you like" }\end{array}$ & Dixon et al ${ }^{35}(2018)$ \\
\hline
\end{tabular}

study design echoes that responsible for the stepwise successes in the late 20th century that produced cures for the deadliest childhood cancer, acute lymphoblastic leukemia (ALL). This disease was successfully treated by modifying diverse evidence-based practices without relying on pharmacologic or other major treatment breakthroughs. Despite this, the effort yielded successful personalized interventions that were not obtainable for other severe childhood conditions..$^{40}$ EPINET hopes to automate much of these stepwise advances with a learning health system. This program relies on data routinely collected in clinical practice to drive the process of scientific discovery. Specifically, it determines the relationships between clinical features, biologic measures, treatment characteristics, and symptomatic and functional outcomes. EPINET aims to accelerate our understanding of biomarkers of psychosis risk and onset, as well as factors associated with recovery and cure. Dashboard displays of outcomes will allow for real-time comparisons within and across early intervention clinics. This in turn identifies performance gaps and drives continuous quality improvement.

\section{Barriers to optimizing program efficacy for both models}

Unfortunately, there are stark differences between ALL and severe mental disorders that potentially jeopardize the achievement of these aims, despite the advances in data analytic abilities that drive the learning health system. Specifically, the heterogeneity of psychotic illnesses and the absence of reliable prognostic and modifiable risk markers (responsible for failed efforts to enhance treatment of serious mental illness over the last half century ${ }^{1,2,41}$ ) are unlikely to be resolved by a learning health system. These measures are vital to determine whether specific interventions are effective, particularly given the absence of a randomized control group in the EPINET/learning health system design. Fortunately, however, the National Institutes for Health has recently initiated the Accelerating Medicines Partnership-Schizophrenia (AMP-SCZ). This approach seeks "promising biological markers that can help identify those at risk of developing schizophrenia as early as possible, track the progression of symptoms and other outcomes and ultimately define targets for treatment development." ${ }^{\prime 2}$ The

\section{Clinical Point}

The effective sizes in FEP studies were small to medium on outcome measures tracking recovery 


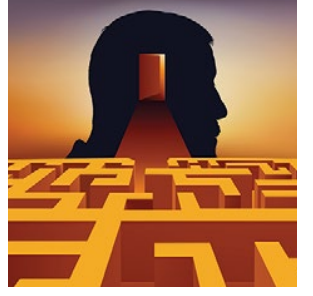

\section{Preventing psychosis}

\section{Clinical Point}

\section{Evidence supports long-acting injectable antipsychotics, early consideration of clozapine, family therapy, and CBT to preserve functioning}

\section{Related Resources}

- Early Assessment and Support Alliance. National Early Psychosis Directory. https://easacommunity.org/nationaldirectory.php

- Kane JM, Robinson DG, Schooler NR, et al. Comprehensive versus usual community care for first-episode psychosis: 2-year outcomes from the NIMH RAISE Early Treatment Program. Am J Psychiatry. $2016 ; 173(4): 362-372$

Drug Brand Name

Clozapine · Clozaril

Box ${ }^{1,4,9,10,36,41,43-45}$ (page 27) describes some of the challenges involved in identifying biomarkers of severe mental illness.

\section{A strength-based approach}

The absence of sufficiently powered RCTs for prevention studies and the reliance on intermediate outcomes for FEP studies leaves unanswered whether such programs can effectively prevent chronic psychosis at a cost society is willing to pay. Still, substantial evidence indicates that outreach, long-acting injectable antipsychotics, early consideration of clozapine, family therapy, CBT for psychosis/attenuated psychosis, and services focused on competitive employment can preserve social and occupational functioning. ${ }^{16,34}$ Until these broader questions are more definitively addressed, it seems reasonable to apply what we have learned (Table, ${ }^{11,12,35,37-39,46}$ page 29).

Simply avoiding the most divisive aspects of the medical model that inadvertently promote stigma and undercut selfconfidence may help maintain patients' willingness to learn how best to apply their strengths and manage their limitations. ${ }^{11}$ The progression to enduring psychotic features (eg, fixed delusions) may reflect ongoing social isolation and alienation. A strength-based approach seeks first to establish common goals (eg, school, work, friends, family support, housing, leaving home) and then works to empower the patient to successfully reach those goals. ${ }^{35}$ This typically involves giving them the opportunity to fail, avoiding criticism when they do, and focusing on these experiences as learning opportunities from which success can ultimately result.

It is difficult to offer all these services in a typical private practice setting. Instead, it may make more sense to use one of the hundreds of early intervention services programs in the United States. ${ }^{46}$ If a psychiatric clinician is dedicated to working with this population, it may also be possible to establish ongoing relationships with primary care physicians, family and CBT therapists, family support services (eg, National Alliance on Mental Illness), caseworkers and employment counselors. In essence, a psychiatrist may be able re-create a multidisciplinary effort by taking advantage of the expertise of these various professionals. The challenge is to create a consistent message for patients and families in the absence of regular meetings with the clinical team, although the recent reliance on and improved sophistication of virtual meetings may help. Psychiatrists often play a critical role even when the patient is not prescribed medication, partly because they are most comfortable handling the risks and may have the most comprehensive understanding of the issues at play. When medications are appropriate and patients with FEP are willing to take them, early consideration of long-acting injectable antipsychotics and clozapine may provide better stabilization and diminish the risk of earlier and more frequent relapses.

\footnotetext{
References

1. Hyman SE. Revolution stalled. Sci Transl Med. 2012; 4(155):155cm11. doi: 10.1126/scitranslmed.3003142

2. Harrington A. Mind fixers: psychiatry's troubled search for the biology of mental illness. W.W. Norton \& Company; 2019 .
}

\section{Bottom Line}

Early interventions for psychosis include the prevention model and the first-episode recovery model. It is difficult to assess, compare, and optimize the effectiveness of such programs. Current evidence supports a 'strength-based' approach focused on finding common ground between patients, their support system, and the treatment team. 
3. Millan MJ, Andrieux A, Bartzokis G, et al. Altering the course of schizophrenia: progress and perspectives. Nat Rev Drug Discov. 2016;15(7):485-515.

4. Lieberman JA, Small SA, Girgis RR. Early detection and preventive intervention in schizophrenia: from fantasy to reality. Am J Psychiatry. 2019;176(10):794-810.

5. McGorry PD, Nelson B, Nordentoft $M$, et al. Intervention in individuals at ultra-high risk for psychosis: a review and future directions. J Clin Psychiatry. 2009;70(9):1206-1212.

6. Csillag C, Nordentoft M, Mizuno M, et al. Early intervention in psychosis: From clinical intervention to health system implementation. Early Interv Psychiatry. 2018;12(4):757-764.

7. McGorry PD, Ratheesh A, O'Donoghue B. Early intervention - an implementation challenge for 21st century mental health care. JAMA Psychiatry. 2018;75(6):545-546.

8. Rosenheck R. Toward dissemination of secondary prevention for psychosis. Am J Psychiatry. 2018;175(5):393-394.

9. Fusar-Poli P, Salazar de Pablo G, Correll CU, et al. Prevention of psychosis: advances in detection, prognosis, and intervention. JAMA Psychiatry. 2020;77(7):755-765.

10. Oliver D, Reilly TJ, Baccaredda Boy O, et al. What causes the onset of psychosis in individuals at clinical high risk? A meta-analysis of risk and protective factors. Schizophr Bull. 2020;46(1):110-120.

11. Tindall $R$, Simmons $M$, Allott $K$, et al. Disengagement processes within an early intervention service for firstepisode psychosis: a longitudinal, qualitative, multiperspective study. Front Psychiatry. 2020;11:565-565.

12. Dixon LB, Holoshitz Y, Nossel I. Treatment engagement of individuals experiencing mental illness: review and update. World Psychiatry. 2016;15(1):13-20.

13. Rickwood D, Paraskakis M, Quin D, et al. Australia's innovation in youth mental health care: The headspace centre model. Early Interv Psychiatry. 2019;13(1):159-166.

14. Woodberry KA, Shapiro DI, Bryant C, et al. Progress and future directions in research on the psychosis prodrome: a review for clinicians. Harv Rev Psychiatry. 2016;24(2):87-103

15. Gupta T, Mittal VA. Advances in clinical staging, early intervention, and the prevention of psychosis. F1000Res. 2019;8:F1000 Faculty Rev-2027. doi: 10.12688/ f1000research.20346.1

16. Correll CU, Galling B, Pawar A, et al. Comparison of early intervention services vs treatment as usual for early-phase psychosis: a systematic review, meta-analysis, and metaregression. JAMA Psychiatry. 2018;75(6):555-565.

17. Cannon TD, Cadenhead K, Cornblatt B, et al. Prediction of psychosis in youth at high clinical risk: a multisite longitudinal study in North America. Arch Gen Psychiatry. 2008;65(1):28-37.

18. Sommer IE, Bearden CE, van Dellen E, et al. Early interventions in risk groups for schizophrenia: what are we waiting for? NPJ Schizophr. 2016;2(1):16003-16003.

19. McGorry PD, Nelson B. Clinical high risk for psychosisnot seeing the trees for the wood. JAMA Psychiatry. 2020;77(7):559-560.

20. van der Gaag M, Smit F, Bechdolf A, et al. Preventing a first episode of psychosis: meta-analysis of randomized controlled prevention trials of 12 month and longer-term follow-ups. Schizophr Res. 2013;149(1):56-62.

21. Marshall M, Rathbone J. Early intervention for psychosis. Cochrane Database Syst Rev. 2011;(6):CD004718. doi: 10.1002/14651858.CD004718.pub3

22. Heinssen RK, Insel TR. Preventing the onset of psychosis: not quite there yet. Schizophr Bull. 2015;41(1):28-29.

23. Amos AJ. Evidence that treatment prevents transition to psychosis in ultra-high-risk patients remains questionable. Schizophr Res. 2014;153(1):240.

24. National Institute for Health and Care Excellence Psychosis and schizophrenia in adults: prevention and management. Clinical guideline [CG178]. 1.3.7 How to deliver psychological interventions. Published February 12, 2014. Updated March 1, 2014. Accessed August 30, 2021. https://www.nice.org.uk/guidance/cg178/chapter/ recommendations\#how-to-deliver-psychologicalinterventions

25. Fusar-Poli P, Werbeloff N, Rutigliano G, et al. Transdiagnostic risk calculator for the automatic detection of individuals at risk and the prediction of psychosis: second replication in an independent National Health Service Trust. Schizophr Bull 2019;45(3):562-570.

26. Fusar-Poli P, Oliver D, Spada G, et al. The case for improved transdiagnostic detection of first-episode psychosis: electronic health record cohort study. Schizophr Res. 2021;228:547-554.

27. Fusar-Poli P. Negative psychosis prevention trials. JAMA Psychiatry. 2017;74(6):651.

28. Cuijpers P, Smit F, Furukawa TA. Most at-risk individuals will not develop a mental disorder: the limited predictive strength of risk factors. World Psychiatry. 2021;20(2): 224-225.

29. Carrión RE, Cornblatt BA, Burton CZ, et al. Personalized prediction of psychosis: external validation of the NAPLS-2 psychosis risk calculator with the EDIPPP Project. Am J Psychiatry. 2016;173(10):989-996.

30. Worthington MA, Walker EF, Addington J, et al Incorporating cortisol into the NAPLS2 individualized risk calculator for prediction of psychosis. Schizophr Res. 2021;227:95-100

31. Koutsouleris N, Dwyer DB, Degenhardt F, et al. Multimodal machine learning workflows for prediction of psychosis in patients with clinical high-risk syndromes and recent-onset depression. JAMA Psychiatry. 2021;78(2):195-209.

32. Simmons MB, Grace D, Fava NJ, et al. The experiences of youth mental health peer workers over time: a qualitative study with longitudinal analysis. Community Ment Health J. 2020;56(5):906-914

33. Devoe DJ, Farris MS, Townes P, et al. Interventions and transition in youth at risk of psychosis: a systematic review and meta-analyses. J Clin Psychiatry. 2020;81(3):17r12053. doi: 10.4088/JCP.17r12053

34. Bosnjak Kuharic D, Kekin I, Hew J, et al. Interventions for prodromal stage of psychosis. Cochrane Database Syst Rev. 2019;2019(11):CD012236

35. Dixon LB, Goldman HH, Srihari VH, et al. Transforming the treatment of schizophrenia in the United States: The RAISE Initiative. Annu Rev Clin Psychol. 2018;14:237-258.

36. Friedman-Yakoobian MS, Parrish EM, Eack SM, et al. Neurocognitive and social cognitive training for youth at clinical high risk (CHR) for psychosis: a randomized controlled feasibility trial. Schizophr Res. 2020;S09209964(20)30461-8. doi: 10.1016/j.schres.2020.09.005

37. Kane JM, Schooler NR, Marcy P, et al. Effect of long-acting injectable antipsychotics vs usual care on time to first hospitalization in early-phase schizophrenia: a randomized clinical trial. JAMA Psychiatry. 2020;77(12):1217-1224.

38. Morrison AP, Pyle M, Maughan D, et al. Antipsychotic medication versus psychological intervention versus a combination of both in adolescents with first-episode psychosis (MAPS): a multicentre, three-arm, randomised controlled pilot and feasibility study. Lancet Psychiatry. 2020;7(9):788-800

39. Chen YQ, Li XR, Zhang L, et al. Therapeutic response is associated with antipsychotic-induced weight gain in drugnaive first-episode patients with schizophrenia: an 8-week prospective study. J Clin Psychiatry. 2021;82(3):20m13469. doi: 10.4088/JCP.20m13469

40. Insel TR. RAISE-ing our expectations for first-episode psychosis. Am J Psychiatry. 2016;173(4):311-312.

41. Tandon R, Goldman M. Overview of neurobiology. In: Janicak PG, Marder SR, Tandon R, et al, eds. Schizophrenia: recent advances in diagnosis and treatment. Springer; 2014:27-33.

42. National Institutes of Health. Accelerating Medicines Partnership. Schizophrenia. Accessed August 30, 2021. https://www.nih.gov/research-training/acceleratingmedicines-partnership-amp/schizophrenia

43. Guloksuz S, van Os J. The slow death of the concept of schizophrenia and the painful birth of the psychosis spectrum. Psychol Med. 2018;48(2):229-244.

44. Christ-Crain M, Bichet DG, Fenske WK, et al. Diabetes insipidus. Nat Rev Dis Primers. 2019;5(1):54.

45. Ahmadi L, Goldman MB. Primary polydipsia: update. Best Pract Res Clin Endocrinol Metab. 2020;34(5):101469. doi: 10.1016/j.beem.2020.101469

46. Early Assessment and Support Alliance. National Early Psychosis Directory. Accessed August 30, 2021. https:// easacommunity.org/national-directory.php

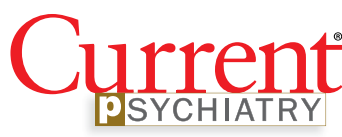

$\nabla^{\text {MDedge.com } / \text { psychiatry }}$

\section{Clinical Point}

Simply avoiding the
most divisive aspects
of the medical model
may help maintain
patients' willingness
to learn how to apply
their strengths

